\title{
A Cost Analysis of Film Image Management and Four PACS Based on Different Network Protocols
}

\author{
David Beard, Deniese Parrish, and Dan Stevenson
}

\begin{abstract}
Picture Archive and Communication Systems (PACS), which allow the electronic acquisition, storage, transportation, and viewing of medical images, hold the eventual promise of reduced costs, improved imagemanagement logistics, and ultimately, improved patient care. But at what point in the future will PACS really cost less than film-based image management for a given hospital size; and how are these costs affected by the choice of the digital communication network? To address these questions, a static differential cost model has been constructed. PAC systems based on two high-speed networks (less then 150 megabytes per second Mbpsl and two lowspeed networks, as well as film, were considered for five different sized hospitals (ranging from 15,000 to 125,000 procedures per year) and two time periods (1995 and 2000). PACS equipment was assumed to have a payoff of five years. The model considered all capital and supply costs and personnel costs for the PACS and for film storage and retrieval. It did not consider any possible cost savings from logistics improvement likely to result from the adoption of a PACS. Based on the assumptions outlined, highspeed-network PACS are less costly than those based on low-speed networks for all scenarios considered. Further, oven though all possible PACS cost savings were not considered, high-speed network PACS appear to be less costly than film for hospitals larger than 60,000 procedures in 1995 and larger than 15,000 in 2000, while low-speed-network PACS should cost less than film for 60,000 and 30,000 procedure hospitals in 1995 and 2000 respectively. (1) 1990 by W.B. Sounders Company.
\end{abstract}

KEY WORDS: PACS, modical imaging, cost analysis.

A PICTURE Archive and Communication System (PACS) is an electronic, and ideally, filmless information system for acquiring, storing, transporting, and electronically displaying medical images. It allows interpretation by radiologists and image viewing by attending

From the School of Medicine, University of North Carolina at Chapel Hill, Chapel Hill, NC.

Supported by National Institute of Health Grants No. ROI CA 44060 and POI CA47982 and by a gift from Bell Northern Research, RTP, NC.

Address reprint requests to David Volk Beard, PhD, Research Division, CB\# 7510 Old Clinic Bldg, University of North Carolina, School of Medicine, Chapel Hill, NC 27599-7510.

(-) 1990 by W.B. Saunders Company.

0897-1889/90/0302-0007\$03.00/0 physicians in other departments. Proponents argue that a PACS will eliminate the need for film and remove the costly physical constraints associated with communication of radiologic image data on film. They believe that electronic images can be more efficiently managed, and simultaneously provided, to a greater number of physicians over a larger geographical area. Unfortunately, such a system is very expensive due to the costs of its hardware and integrating its various components. PACS technology costs, most likely, will continue to plummet in the coming decade, while film costs will continue to rise. Therefore, at what point in the future will a viable PACS cost less than an equivalent film system for a given size hospital?

The cost of PACS will come from the acquisition, implementation, and ongoing maintenance of the system. PACS acquisition costs include those for mass storage devices and related software, digital acquisition devices, hardcopy units, radiology and attending-physician workstations, and communications networks. PACS implementation costs include initial training of personnel, the time and cost of integrating the PACS with the Radiology Information System (RIS), the potential increased requirements for electrical power and cooling, and other relevant setup costs. PACS' ongoing costs include additional training of personnel due to turnover, depreciation and financing of equipment, labor, supplies, the cost for any remaining film requirements, and the replacement of worn or outdated equipment.

The primary cost savings of a fully implemented PACS over film may come from two main factors: first, the almost total elimination of film costs, including the cost of developers, storage for film and chemicals, space for developing machines and personnel time; and second, the more efficient utilization of employees, eg technologists, who no longer have to leave their patients to process films, in institutions like the University of North Carolina (UNC), or the complete elimination of filming technicians at other institutions. At UNC, the time required to print CT and MRI studies to film will be eliminated as 
well. Additional cost savings will occur from elimination of staff manning the current filmbased image management service, darkroom technicians, and possibly radiologists who no longer have to over-staff low-volume remote reading rooms. We anticipate that for some time, even in a completely digital department, physicians will continue to want some hardcopies of images. Eventually this final use of hardcopy should disappear.

Costs for the film system can be divided roughly into nondifferential costs, film differential costs, and PACs differential costs. Nondifferential costs consist of equipment, personnel, and other costs that will not change when hospitals switch from film-based image management to PACS. Film differential costs include yearly film and film supply costs, hardware costs for film developers, space costs for the vast image libraries, and film librarians. PACS differential costs include PACS hardware and maintenance costs. There are many potential PACS logistics cost savings due to the fact that digitized images, unlike film, can be simultaneously in two places. ${ }^{1}$ However, these cost savings are very difficult to quantify. They include reduced CT technologists' time because digitally acquired images no longer have to be printed, and improved radiologist productivity because radiologists can share workload among remote reading areas.

Previous PACS cost analysis work has examined PACS and film costs for single sized hospitals, single communications networks, and single points in time. ${ }^{2-4}$ Seshadri estimated the costs for film and two versions of PACS using cost and procedure volume data from the University of Pennsylvania Department of Radiology. He estimated that a film-based image management would cost $\$ 2.5$ million, a PACS based on digitizing films generated with current radiograph equipment would cost $\$ 2.3$ million, and a PACS using all digital sources and no film would cost $\$ 1.8$ million. The first two scenarios took into account cost savings from discarded film. PACS equipment was depreciated from between six and eight years. Cost analysis of PACS field trials have also been conducted. ${ }^{5}$

Our study looked at PACS/film differential costs. PACS/film differential costs will vary with the size of the systems, measured by the number of workstations and other equipment. Therefore, five generously sized PACS were considered. In looking at historical data on emerging high technology, costs have usually decreased by $50 \%$ every five years. We have assumed that PACS costs will follow that same track and the costs of existing film systems will continue to rise with inflation. Therefore, PACS/film relative cost decisions were considered for two future time periods. Finally, four PAC Systems, each based on different network technologies, were considered in addition to a film-based system.

Section 2 summarizes the hypothetical PAC systems we modeled. Section 3 describes the cost model itself including all cost assumptions. Finally, sections 4 and 5 present the results and conclusions of this research. The Appendix contains addition details of the hypothetical PACS specifications.

\section{HYPOTHETICAL PACS}

A PACS can mean anything from a few workstations and some disks to millions of dollars of high technology hardware and software. Therefore, a PACS cost analysis must be based on a precise definition of the hypothetical PACS technology to be modeled. The following is a summary of the detailed PACS descriptions and costs given in the Appendix. PACS and film cost assumptions are in Table 1 .

We have found that network speed is a significant factor in total PACS cost. Low-speed (LS) image communications networks, with a sustained rate of less than 100 megabits per second (Mbps) to each node, cannot transmit images fast enough to provide sufficient response time for viable medical image-workstation interaction. Thus LS medical image workstations must have sufficient main memory, swap space, bus bandwidth, and central processing unit (CPU) speed to store the entire patient image folder local to the workstation, making each workstation very expensive. All current PACS, and all current PACS cost analyses, are based on lowspeed-network technology. High-speed (HS) networks, with a sustained rate of $150 \mathrm{Mbps}$ or more to each node, can transmit images with sufficient speed to keep up with worse-case medical image workstation interactions. The HS workstation thus can be reduced to framebuffers, monitors, and a small controlling CPU. The workstation cost savings for high-speed networks are some- 
Table 1. Cost and Size Estimates for a 100,000 Procedure System in 1995

\begin{tabular}{|c|c|c|c|c|c|}
\hline & Film Nos. & PACS Nos. & $\begin{array}{c}\text { Cost } \\
\text { of Film (\$) }\end{array}$ & $\begin{array}{c}\text { Low Speed Cost } \\
\text { Per Unit } \\
\text { (Ether Net) }\end{array}$ & $\begin{array}{l}\text { High Speed Cost } \\
\text { Per Unit } \\
\text { (BISDN) (\$) }\end{array}$ \\
\hline \multicolumn{6}{|l|}{ Labor costs } \\
\hline Developer Maintenance & 0.4 & 0 & 39,565 & 39,565 & 39,565 \\
\hline Film clerks & 13 & 1 & 29,394 & 29,394 & 29,394 \\
\hline Film supervisors & 1 & 0 & 35,273 & 35,273 & 35,273 \\
\hline PACS operators & 0 & 1 & 0 & 29,394 & 29,394 \\
\hline PACS maintenance & 0 & 2 & 0 & 57,433 & 57,433 \\
\hline PACS supervisor & 0 & 1 & 0 & 63,814 & 63,814 \\
\hline \multicolumn{6}{|l|}{ Film equipment } \\
\hline Developers & 16 & 1 & 25,536 & 25,536 & 25,536 \\
\hline Alternators & 16 & 0 & 12,763 & 12,763 & 12,763 \\
\hline \multicolumn{6}{|l|}{ Maintenance rate on film equip- } \\
\hline \multicolumn{6}{|l|}{ Film supplies } \\
\hline Film costs & & & 573,062 & 30,161 & 30,161 \\
\hline Film supplies & & & 654,915 & 34,469 & 34,469 \\
\hline Silver recovery & & & $(63,814)$ & $(3,359)$ & $(3,359)$ \\
\hline \multicolumn{6}{|l|}{ PACS equipment } \\
\hline Hard-copy devices & 0 & 1 & 0 & 35,000 & 35,000 \\
\hline Plate digitizers & 0 & 5 & 0 & 70,000 & 70,000 \\
\hline Film digitizers & 0 & 2 & 0 & 40,000 & 40,000 \\
\hline Super RWS & 0 & 8 & 0 & 88,672 & 38,574 \\
\hline RWS & 0 & 20 & 0 & 34,030 & 12,272 \\
\hline APWS & 0 & 88 & 0 & 10,510 & 1,753 \\
\hline Archive & 1 & 1 & 0 & 177,000 & 209,000 \\
\hline Network base cost & & & 0 & 1,000 & 200,000 \\
\hline Network per-node cost & & & 0 & 250 & 5,000 \\
\hline Network wiring cost per node & & 0 & 0 & 2,176 & 1,276 \\
\hline \multicolumn{6}{|l|}{ Maintenance rate on PACS equip- } \\
\hline \multicolumn{6}{|l|}{ Space } \\
\hline Space per developer & 90 & 90 & & & \\
\hline Film room space & 1400 & 0 & & & \\
\hline Space per digitizer & 20 & 20 & & & \\
\hline Space per hard copy & 10 & 10 & & & \\
\hline Archive space & 100 & 100 & & & \\
\hline Space cost per sq ft & & & 210 & 210 & 210 \\
\hline Total space cost & & & 16,170 & 3,216 & 3,216 \\
\hline Inflation rate $\quad 5 \%$ & & & & & \\
\hline Interest rate on capital & & & & & \\
\hline
\end{tabular}

Worse-Case PACS prices are $100 \%$ higher than those listed.

PACS prices are assumed to decrease by $50 \%$ every 5 years.

Workstation software costs $(\$ 510,000)$ are divided by number and cost among all the workstations of five 100,000 procedure hospital systems.

Workstation costs do not include marketing costs or profit because workstations may be sold as "lost leaders" to other network or radiologic equipment. Adding marketing or profit costs would likely double the stated workstation costs, and would roughly correspond to the worse-case scenario.

34 additional network nodes will be needed in addition to those listed above.

what offset by the more costly archive required to be able to keep up with the network data rates and by the additional cost of the faster communication network. While high-speed networks will become commercially available by 1993 , there is no guarantee that any PAC based on that technology will become commercially available by 1995 .
Because of the uncertainty surrounding HS PACS availability, we have modeled future PACS costs based on two low-speed networks, EtherNet and the current version of the Fiberoptic Distributed Data Interface (FDDI), and two high-speed networks Broadband Integrated Services Digital Network (BISDN) and the High Performance 
Parallel Interface (HPPI), formally known as the High-Speed Channel. BISDN (available between 1992 and 1995) is particularly advantageous for two reasons: first, because as much as the full 150 Mbps can be dedicated to each user without interference with resources available to other network users, and second, because it has a downtime requirement of only two hours every 40 years. Because the costs and results for FDDI and EtherNet, and for HPPI and BISDN are so similar, we have simplified our presentation by describing only the "Low-Speed (LS)" and "High-Speed (HS)" PAC Systems.

We addressed the cost of converting plain film to digital format with one of the radiograph plate technologies. This new technology is important to PACS, because it allows images from existing radiograph devices, including portables devices, to be included in a complete PACS without the use of any film or replacement of nondigital acquisition devices. We assume that adequate image quality could be obtained with this technology by 1995 .

Electronic workstations are the essential means for physicians to view medical images. For the purposes of our cost analysis, we have assumed three types of image viewing workstations ${ }^{6.7}$ : a super radiology workstation (SRWS), a radiology workstation (RWS), and an attending physician workstation (APWS). The SRWS, with three $2,000^{2}$ screens would be used for the primary diagnosis. It has both $3 \mathrm{D}$ and image processing capacity. The RWS, with a single $2,000^{2}$ screen, would be used for less demanding diagnoses. The APWS, with a single $1,000^{2}$ screen, would be used by attending physicians to review images. Workstation costs and numbers are given in Table 1, with details in the Appendix.

The advent of optical data devices that can store and quickly access vast quantities of image data is one of the critical technological advances that makes PACS feasible. Optical jukeboxes ${ }^{8}$ that allow access to ten or more data platters, each holding up to seven gigabytes, are available as are optical tape drives capable of storing one to three terabytes completely scanned in under one minute. High-speed compression and decompression hardware is available that allows between $3: 1$ and $4: 1$ compression while mathematically guaranteeing the original image upon de- compression. Such optical image storage devices, combined with conventional disk and main memory storage technology, can produce a twolayered or "virtual memory" storage system capable of reliably storing seven or more years of image data while still allowing very fast access to almost all patient data and images. We estimate that the equivalent of about two terabytes of images are generated by a hospital performing 100,000 procedures per year. While the hypothetical low-speed-network archive can simply consist of a large jukebox, the hypothetical highspeed-network archive, because of its requirement to keep up with the very fast network data rates, must have both a long-term and high-speed short-term memory. The long term memory can consist of a teribyte optical tape drive. The short term memory, consisting of many small disk drives operating in parallel, can provide a sufficient output rate to drive all the workstations with adequate response time.

\section{COST MODEL}

We have constructed a simple differential cost model of both film-based image-management and four different PACS. We consider 1995 to 1999 and 2000 to 2004 time periods, with most costs initially developed from the 1990 base-line year. Our model includes film and PACS costs for four hypothetical hospitals in addition to $\mathrm{UNC}$ as measured by the number of procedures generated per year $(15,000,30,000,60,000$, 100,000 , and 125,000 ). While initial equipment configuration estimates were based on fractions of estimates for UNC (125,000 procedures per year), these were confirmed with data from other hospitals of appropriate sizes. The final model consists of 33 integrated spreadsheets. Model assumptions are presented in Table 1 .

For the last twenty years, high-technology computer hardware, upon which PAC are based, has been decreasing in price (or increasing in performance) at about $50 \%$ every five years. An assessment of emerging computer technology indicates that this trend will continue for at least the next ten years. One could also estimate that prices would remain the same with performance increasing by $50 \%$ in five years, but we have chosen to do our costing at a fixed performance level. Based on this, we have determined PACS component costs for 1995 and 2000 by estimat- 
ing what computer-human-interaction and other performance requirements would be required, determining the cost of meeting those requirements using 1990 technology prices, and assuming the 1990 prices would be reduced by $50 \%$ in 1995 and by an additional $50 \%$ in 2000 . Details of PACS component specification and pricing are given in the appendix.

This model compares the differential acquisition costs of both film and PACS. Since we are not considering any changes in radiologist productivity, radiologist salaries and time are not included in this analysis. Acquisition costs include all the differential costs for initially acquiring either a PACS or a film system. Thus when costing film image management, we value various hardware in terms of the probable initial purchase price, rather than the residual value when sold after PACS acquisition.

We model both PACS and film in steady state, not during the film to PACS transition period. Thus, for example, we ignore initial training and only consider ongoing training costs, which are included in the annual personnel salaries. We assume that the system has been operational for several years before modeling, and that the only remaining undigitized films are those in the remote warehouse. Inflation and maintenance contracts are included. Average-case and worsecase costs are modeled, with worse-case prices being about $100 \%$ higher than average-case.

Most film costs are periodic, that is, they reoccur each year, while most PACS costs are capital, occurring once at purchase. Therefore, for comparison, we assumed a five year payoff period and generated three PACS and two film costs figures, eg total PACS capital costs, average annual differential costs during-payoff, and average annual differential costs post-payoff. Total capital cost is the best measure for comparing the costs of the various PACS. Average annual costs during- and post-payoff are the best measures for comparing PACS and film. Both during- and post-payoff costs are included to avoid the confusion of determining the residual value of capital equipment after depreciation.

Several factors besides hardware choice and numbers effect the cost of a PACS including maintenanc contracts, labor, supplies, and space. We assume that both on-site personnel and maintenance contracts will be required for PACS equipment. Labor costs listed in Table 1 include both salaries and fringe benefits. Equipment maintenance contracts are assumed to cost $10 \%$ of capital purchase price per year for the archive, network, hardcopy devices, digitizers, and workstations, with additional personnel available for PACS maintenance. Maintenance contracts for film equipment were assumed to be $5 \%$ of purchase price per year, with an additional 0.5 full-time employees for routine developer maintenance. PACS supplies include optical tapes or disks, film related supplies for the hardcopy devices, and replacement digital phosphor plates. Space requirements listed in Table 1 include space for all equipment footprints. Purchase costs of film developers and alternators are included in the cost of the film system. Viewboxes are ignored due to their small cost.

\section{Results}

Figure 1 shows the average annual differential point costs during the five year payoff period for film, the low-speed systems, and the high-speed systems, purchased either in 1995 or in 2000 . To resummarize the assumptions stated above, PACS differential costs include all PACS equipment and any additional PACS related labor, for example, the archive operator. Interest on the payoff debt is assumed to be $10 \%$. Film differential costs include yearly film and supply costs, personnel costs for those individuals who clearly would be eliminated with PACS acquisition, such as most of the film vault staff, and any equipment that would likely be eliminated with PACS' acquisition such as many film developing machines.

Figure 2 shows the average annual differential costs after the five year payoff period. Depreciation rates are difficult to anticipate and require

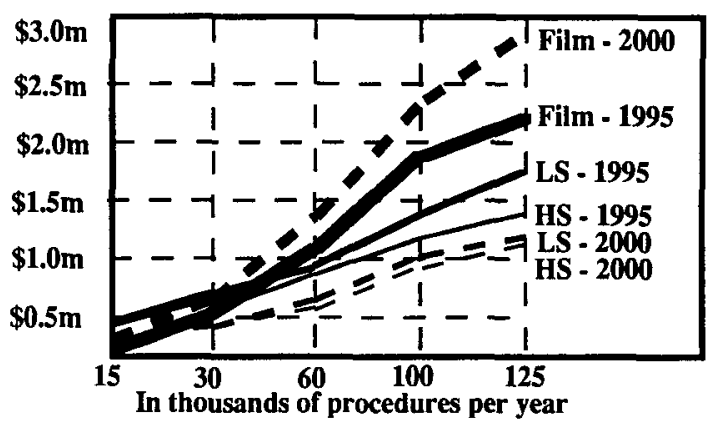

Fig 1. Average annual differential costs during payoff. 


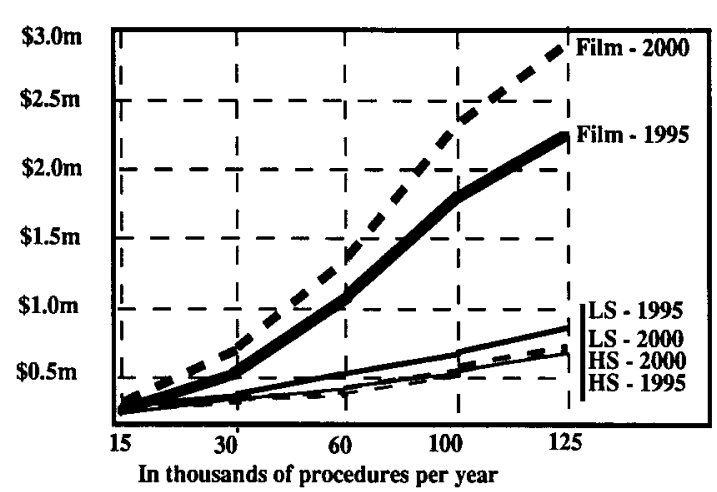

Fig 2. Average annual differential costs after payoff.

estimating the residual value of each piece of PACS and film equipment. Instead we have chosen to present both the annual cost during and after the five year payoff period to allow the reader to informally apply his or her own notions of equipment residual life. Note that the difference between 1995 and 2000 costs are due to a $50 \%$ reduction in high-tech hardware costs, offset by inflation (5\% per year for five years) for all other costs. Note that the main difference between LS and HS post payoff costs is due to maintenance on the different priced hardware. Figure 3 shows capital costs for LS and HS PACS in 1995 and 2000. Note that purchase year appears more significant than network speed.

Table 2 shows LS and HS capital costs for three variations of 100,000 procedure systems purchased in 1995 and in 2000. It illustrates the significance of the number of workstations and PACS equipment prices on total system cost. "Average" uses the equipment costs given in Table 1. "Worse" uses $100 \%$ higher prices.

Workstation numbers are also considered. The first row details a 100,000 procedure system from which all medical image workstations have been eliminated. The second row describes the stan-

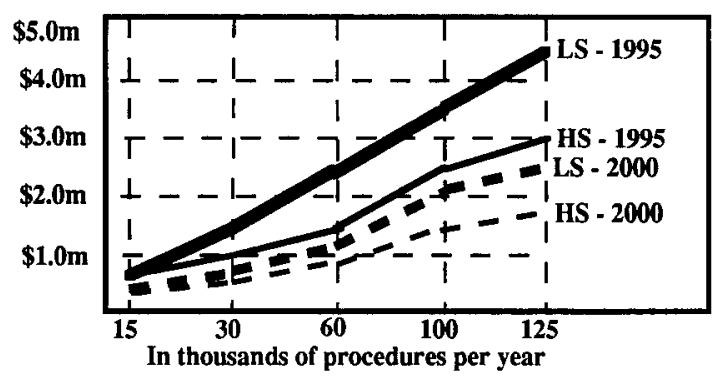

Fig 3. Capital costs for low speed and high speed PACS.

\begin{tabular}{|c|c|c|c|c|}
\hline & \multicolumn{2}{|c|}{ Low Speed } & \multicolumn{2}{|c|}{ High Speed } \\
\hline & Average & Worse* & Average & Worse" \\
\hline $\begin{array}{l}\text { No. workstations } \\
8 \text { SRWS, } 20 \text { RWS, }\end{array}$ & $\$ .85 \mathrm{~m}$ & $\$ 1.5 m$ & $\$ 1.1 \mathrm{~m}$ & $\$ 1.8 \mathrm{~m}$ \\
\hline $\begin{array}{l}88 \text { APWS } \\
16 \text { SRWS, } 40 \text { RWS, }\end{array}$ & $\$ 3.7 \mathrm{~m}$ & $\$ 6.7 m$ & $\$ 2.6 \mathrm{~m}$ & $\$ 4.0 \mathrm{~m}$ \\
\hline 176 APWS & $\$ 6.7 \mathrm{~m}$ & $\$ 12.0 \mathrm{~m}$ & $\$ 4.1 \mathrm{~m}$ & $\$ 6.2 \mathrm{~m}$ \\
\hline
\end{tabular}

-100\% higher PACS equipment prices.

dard 100,000 procedure system. The third row details the system with the number of workstations doubled. Because of the large initial cost for the network switch and the faster archive, the nonworkstation LS system is less than that of the nonworkstation HS system. Note also that workstation costs are from $1 / 2$ to $3 / 4$ of total system cost. In general, as the number of workstations increase, the price advantage of the high-speed system increases. Figure 4 shows average annual differential costs during payoff for film and PACS based on worse-case PACS equipment costs.

\section{CONCLUSIONS AND DISCUSSION}

Based on our assumptions, we draw the following conclusions: (1) A high-speed PACS will cost less than a low-speed PACS for all hospitals generating at least 15,000 procedures per year starting in 1995. It is important to note that while the largest hospital we consider only generates 125,000 procedures, the HS cost advantage increases with the number of workstations, so the conclusions should hold for larger hospitals. (2) A HS PACS will cost less than film for all hospitals generating at least 60,000 procedures

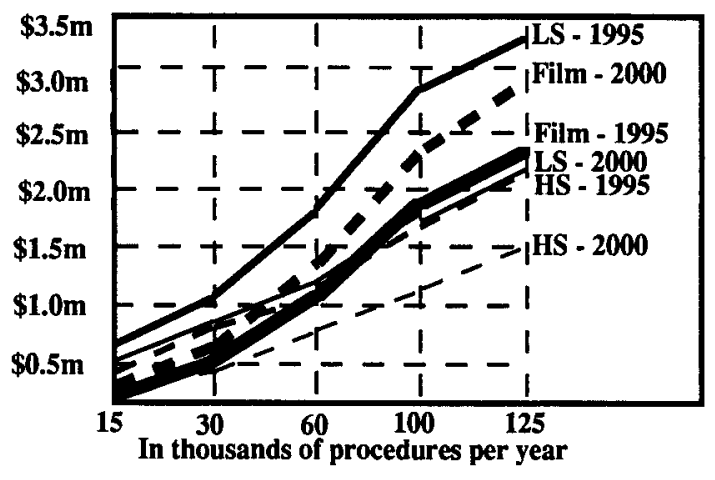

Fig 4. Average annual differential costs during payoff using worse case (+100\%) PACS equipment costs. 
per year if purchased in 1995, and for hospitals generating at least 15,000 procedures if purchased in 2000. (3) A LS PACS will cost less than film for hospitals generating at least 60,000 procedures in 1995 and 30,000 procedures if purchased in 2000. (4) Post payoff costs for both HS and LS PACS range from almost the same as film for 15,000 procedure systems in 1995 , to an annual advantage of $\$ 2.3$ million over film in 2000 for a 125,000 procedure system.

These results are fairly robust relative to equipment numbers and costs. For example, the 125,000 HS PACS system costs less than the 100,000 film system in 1995 , and the $100,000 \mathrm{HS}$ PACS system costs less than the 60,000 Film system in 2000. Even with doubling PACS equipment costs, PACS still costs less than film for 100,000 procedure or larger hospitals, even in 1995.

In general, technology cost projections must be taken with a grain of salt, and this is the case with our analysis. Estimates of cost, number, and performance requirements of medical image workstations may be of limited accuracy, plainfilm-resolution requirements may be understated, and while BISDN and HPPI technologies are (or will become) commercially available, vendors may not have marketed the corresponding high-speed radiology workstations by 1995 .

Nevertheless, there are several clear trends that this cost analysis demonstrates, which transcend the cost and size data: First, applying sensitivity analysis to our cost model shows that workstation cost and numbers are among the two most significant variables for PACS differential costs. Productivity improvements may show labor savings to be also very significant, but productivity is beyond the scope of this analysis. Second, even with the increased cost of the archive and switch, the high-speed-network PACS has a cost advantage over the low-speed system, and that advantage increases with the size of the hospital and number of workstations. Thus workstations and archives for a high-speed-network PACS should be explored and developed. Third and most importantly, PACS can be cost-effective within the next five to ten years for many larger hospitals.

\section{ACKNOWLEDGMENT}

We gratefully acknowledge our colleagues Steve Pizer, $\mathbf{R}$. Eugene Johnston, Robert Cromartie, both for work on which this paper is based and for useful conversations. Our radiologist colleagues, J. Randolph Perry, William McCartney, Jeffrey Creasy, and Paul Jaques, have provided a critical contribution of the clinical issues of display requirements. We thank Terri Bass and Joy Telle for help with the manuscript.

\section{APPENDIX}

\section{Details of Hypothetical PAC Systems}

Several critical components are required to produce an adequate PACS: digital acquisition of all image modalities; film digitizers which can transform existing film-based images into a digital form; hardcopy devices that allow images to be moved to non-PACS institutions; digital communications networks that can move images at extremely high speeds; radiology workstations which allow radiologists to interpret images, produce reports, and consult with attending physicians; and mass storage devices that can store and quickly access many teribytes of data.

\section{Image Acquisition and Hardcopy}

Images can be acquired from several digital sources including MRI, CT, and nuclear medicine. We included network ports for each of these sources in our analysis. Digital radiographs are addressed with the digital radiograph plate, a new technology which saw field testing in 1989. With this technology, a $14 \times 17$-in phosphor plate is placed into a standard film holder and exposed to $\mathrm{x}$-rays in a conventional or portable radiograph machine. Then instead of being developed in a film processor, the film holder is placed into an automated digitizer and digitized at about 2,048 12 -bit pixel resolution. The theoretical resolution limitations of the phosphor plate are sufficient that the plate could be digitized at five line pairs per millimeter, if needed, a resolution possibly required for mammograms. This new technology is important to PACS because it allows images from existing radiograph devices, including portables devices, to be included in a complete PACS without the use of any film or replacement of nondigital acquisition devices. We estimate that a 100,000 procedure hospital would require about five of these units. Our cost analysis assumed that the digital plates would all but eliminate film use. While we assume that the active film library is completely digital, it is likely that films will remain in the remote warehouse for many years and that films will continue to arrive from other institutions. Therefore, we included two film digitizers to provide the needed coverage.

Since a radiology department or hospital may acquire a PACS before the rest of the hospital or other hospitals, the PACS must have the ability to generate conventional film from all images. Thus, in the foreseeable future, any PACS must have hardcopy devices, along with the film associated costs of film, supplies, and developers. We estimate that with the low demand for hardcopy with a complete PACS system, only one unit would be required for a 100,000 procedure hospital.

\section{PACS Communications}

A second component of a PACS is the image communications network, which can move images from their acquisition devices to mass storage, and from mass storage to the radiology and attending physician workstations. For the 
purposes of our analysis, we divide the communication network world into two halves: low-speed (LS) and highspeed (HS) networks. While several tele-radiology devices are on the market that allow images to be transmitted over phone lines at 9,600 baud, they are completely inadequate for even the smallest PACS.

Low-speed networks. Low-speed networks, such as EtherNet and The Fiberoptic Distributed Data Interface (FDDI), provide less than $150 \mathrm{Mbps}$ to each network node. EtherNet comes in several variations: $3 \mathrm{Mbps}$ and $10 \mathrm{Mbps}$ data rates, twisted pair, or coax options. It is well suited for terminal to host text oriented communications. Its performance is not consistent with the needs of image transfer where speed is a consideration. We estimate that in 1995, EtherNet will cost about $\$ 250$ per node with an additional $\$ 1,000$ in overall costs. FDDI, a private network solution promoted by the computer industry, is a technology for Metropolitan Area Networks. It features shared media technology based on principles of token exchange on a ring structurc. It provides $100 \mathrm{Mbps}$ of bandwidth to be shared among all users. The technology supports a maximum ring diameter of $100 \mathrm{~km}$ although there are bandwidth reach tradeoffs reported ${ }^{10}$ Use of fiber transport results in low EMI and a low bit errar rate. ${ }^{10.12}$ We estimate that in 1995, FDDI will cost about $\$ 5,000$ per node with an additional $\$ 30,000$ in additional costs. ${ }^{11}$

High-speed networks. High-speed networks provide 150 or more Mbps to each node on the network. They have been considered strong candidates for PACS communication. ${ }^{12}$ Broadband Integrated Services Digital Network (BISDN) is a high performance networking standard and Wide Area Network technology compatible with standard digital fiberbased long-haul transport and is promoted by the telecommunications industry. Standards are expected to be finalized in the 1991 to 1992 time frame. It features a dedicated 150 Mbps access to each user utilizing a star-oriented architecture with central-office or PBX based switch hubs. As much as the full access rate can be dedicated to each user without interference with resources available to other network users. This eliminated the performance degradation other networks experience with increasing numbers of nodes. The current consensus in the standards forums is that BISDN will be based on Asynchronous Transfer Mode (ATM) technology. ATM is essentially a packet oriented capability which features fixed size cells, 48 bytes of data and 5 bytes of header. It is capable of meeting the diverse needs of voice, data, and video communications on a single network technology consisting of switches, access, transport, and network management infrastructure. The lost packet rate is $10^{7}$ or better and sequential delivery is inherent. Anticipated availability for ATM is between 1992 and 1995. It appears well suited for image applications. In 1995, we estimate that BISDN will cost about $\$ 5,000$ per node with an additional $\$ 200,000$ for the high-speed-network switch. The large switch cost could be shared among several small hospitals or clinics, though we have done all our cost analysis assuming that each hospital would purchase its own BISDN PBX switch. Because BISDN is designed for telephone applications with a down time requirement of 2 hours in $\mathbf{4 0}$ years, this reliability should be particularly useful for medical image applications.

High Performance Parallel Interface (HPPI), formally known as the High-Speed Channel is a proposed ANSI standard for a breathtakingly fast communications interface. It is based on the Cray HSX channel connect design and comes in two versions, 800 Mbps and 1.6 gigabit per second (Gbps). It is intended to support visually oriented supercomputer applications. The standard is currently centered around a copper interface that is inherently very reach limited ( 25 meters) and is suited for application in a computer room. Fiber-based interfaces are beginning to be discussed as part of the standards process. This will result in extended reach for HPPI. Recently products have appeared on the market using fiberoptic technology that provides a reach of $2 \mathrm{~km}$. The interface standard is oriented toward point to point communications and does not inherently support networked communications. Experimental networking capabilities based on HPPI have been proposed and developed. None of these are commercially available. One vendor, Ultranet, ${ }^{13}$ has products available that have HPPI interface options. Ultranet is a hub oriented gigabit per second local area network (LAN) technology based on wide parallel datapath bus architecture. The HPPI interfaces utilized with Ultranet LAN technology will have the same reach limitations inherent in HPPI itself. Lower speed hub interconnect options are available for networking together multiple Ultranet hubs. These options provide $100 \mathrm{Mbps}$ interconnect speeds with reach limited to a few kilometers. Such arrangements may be suitable for PACS applications with properly designed network topologies. We project that in 1995, HPPI should cost about $\$ 9,500$ per node with an additional $\$ 50,000$ in overall costs.

Estimating wiring costs for a cost analysis is difficult because hospitals may or may not already have conduit in place in which to run the fiber. Since most radiology departments are adapting computerized radiology information systems (RIS) we assume that conduits already exist, and that PACS must only include the cost of the actual supplies and labor to run and connect the cables. We assumed a wiring cost of about $\$ 1,000$ per node in 1995. Given the large capital costs of a PACS system, the inclusion of conduit costs would not significantly affect the overall results of the cost analysis.

\section{Medical Image Workstations}

Workstation specification. Electronic workstations are the essential means for physicians to view medical images. For the purposes of our cost analysis, we have assumed three types of image viewing workstations ${ }^{6.7}$ : first, a super radiology workstation (SRWS) with three 2,048 ${ }^{2}$ display screens, 3D, and image processing capability, a radiology workstation (RWS) with one $2,048^{2}$ display screen, and a attending physician workstation (APWS) with a single 1,024' display screen. The user must be able to scroll these screens to view the next set of images in the patient folder with sufficient speed to avoid loosing mental context or becoming frustrated. Our experience with a single $1,024^{2}$ prototype workstation $n^{7.14}$ indicates that a response time which replaces $1 / 2$ the screen's contents in 0.5 seconds is good, in 1.0 seconds is marginally acceptable, and in 2.0 seconds is occasionally acceptable. This translates into a burst data rate requirement for the attending physician workstation of $16 \mathrm{Mbps}$, half a 1,024: image with 12 to 16 bit pixels in 0.5 seconds, and a radiology workstation requirement of $64 \mathrm{Mbps}$. The larger the display area the longer the viewing time, and the less critical a 
super-fast response time. Therefore, we believe that a super radiology workstation will require $128 \mathrm{Mbps}$. Our experience with our prototype workstations indicates that on average, a scroll operation will occur every 2 seconds under worse-case conditions. All archive cost estimates are determined by pricing the lowest cost 1990 configuration that would meet the required performance. All workstation cost estimates include both the cost of the 1990 hardware configuration and four man years of software development, which is estimated to be $\$ 510,000$ in 1995 , spread among the workstations of five 100,000 procedure hospital systems, and prorated to each workstation by price and numbers. Using this costing method, software costs amount to about $10 \%$ of total workstation cost. 1995 prices are a $50 \%$ reduction of 1990 hardware prices plus software costs.

Low-speed workstations. Low-speed image communications networks such as EtherNet and FDDI cannot transmit images fast enough to provide sufficient response time for a scroll operation. Thus radiology workstations designed for low-speed networks must have sufficient main memory, swap space, bus bandwidth, and CPU speed to store the entire patient image folder local to the workstation, making them very expensive. We estimate that an EtherNet super radiology workstation with three $2,048^{2}$ displays and image processing capability would cost $\$ 88,672$ in 1995 , a radiology workstation with a single $2,048^{2}$ display would cost $\$ 34,030$, and a attending physician workstation would cost $\$ 10,510$.

High-speed workstations. High-speed networks can transmit images with sufficient speed to keep up with the scroll operation, thus eliminating the need for large virtual memories, high-speed busses, and fast CPUs. The workstation configuration is reduced to frame buffers and a small controlling CPU. We estimate that in 1990 , a super radiology workstation (SRWS) with three $2,048^{2}$ displays would cost $\$ 38,574$, a radiology workstation (RWS) would cost $\$ 12,272$, and an attending physician workstation (APWS) would cost $\$ 1,753$. Network port costs for each workstation are not included in workstation costs; they are factored into the cost analysis elsewhere.

Workstations for use with high-speed networks are considerably less expensive than those for low-speed networks. Given the large number of workstations a 100,000 procedure hospital requires, this workstation cost difference has a considerable impact on total PACS capital costs. However, these workstation cost savings for high-speed networks are somewhat offset by more costly archives, required to be able to keep up with the network data rates as described below.

Workstation numbers. We estimated the required number of SRWS, RWS, and APWS workstations for a UNC sized PACS system by counting the number of locations where radiologists and attending physicians often view images. At UNC, there are about 20 locations in the radiology department for primary interpretation of medical images that would require either a SRWS or a RWS. Further, emergency rooms, operating rooms, and intensive care units all would require RWSs. Finally, physicians may be at many other locations, including their offices, when they need to view small numbers of images. Based on this second approach, we estimate that a 100,000 procedure hospital would be able to provide adequate patient care with about 8 SRWSs, 20 RWSs, and about 88 APWSs. Workstation-number estimates for these other hospital sizes were estimated from per-procedure requirements derived for UNC.

\section{Image Archives}

Data requirements. An extremely large amount of image information is generated each year. We estimate that the equivalent of 2.5 tbyte of images are generated annually at UNC, depending on the hypothesized digitized resolution of plain film and the average size of each type of study. This amounts to about 2 tbyte per year for a hospital generating 100,000 procedures per year (Table 3 ). This figure is similar to other independently generated estimates. ${ }^{15} \mathrm{We}$ assume that old images must remain on file for at least 7 years, with longer periods required for pediatric cases, for a total image-archive size of about 15 tbyte for a 100,000 procedure hospital. With the current film system, it is rare for a folder to be requested after about 1.5 years of inactivity. However, with the improved image response time of a PACS, this access pattern is likely to change.

Text information such as patient records and scheduling, are increasingly being stored and managed electronically using a Radiology Information System (RIS). Such a system provides fast access to patient records and procedure requisitions, automatically schedules procedures, automatically requests patient folders from either a film-based image management service (IMS) or a PACS, and gathers billing

Table 3. 1990 UNC Image Generation if Digitized

\begin{tabular}{|c|c|c|c|c|c|c|c|}
\hline \multirow[b]{2}{*}{$\begin{array}{l}\text { Procedure } \\
\text { Type }\end{array}$} & \multirow[b]{2}{*}{$\begin{array}{c}\text { No. } \\
\text { Procedure }\end{array}$} & \multirow[b]{2}{*}{$\begin{array}{l}\text { No. } \\
\text { Films }\end{array}$} & \multicolumn{3}{|c|}{ UNC Cases } & \multicolumn{2}{|c|}{$\begin{array}{c}\text { Megabyte per } 100,000 \\
\text { Procedures }\end{array}$} \\
\hline & & & $\begin{array}{c}\text { Size } \\
\text { (Mbyte) }\end{array}$ & $\begin{array}{l}\text { Megabyte/ } \\
\text { Procedure }\end{array}$ & $\begin{array}{c}\text { Total } \\
\text { (Mbyte) }\end{array}$ & Size & Total \\
\hline Large Films & 46,245 & 2.1 & 7.50 & 15.75 & 728,359 & 37,029 & 583,200 \\
\hline Small Films & 16,001 & 3 & 1.50 & 4.5 & 72,005 & 12,812 & 57,654 \\
\hline Portables & 29,650 & 1.5 & 7.50 & 11.25 & 333,563 & 23,741 & 267,085 \\
\hline Fluoroscopy & 5,717 & 8 & 7.50 & 60 & 343,020 & 4,578 & 274,658 \\
\hline Augiography & 1,667 & 40 & 7.50 & 300 & 500,100 & 1,335 & 400,433 \\
\hline Ultrasound & 9,308 & 16 & 0.25 & 40 & 372,320 & 7,453 & 298,118 \\
\hline Nuclear medicine & 3,215 & 32 & 0.03 & 1 & 3,215 & 2,574 & 2,574 \\
\hline CT-Head & 6,048 & 20 & 0.38 & 7.5 & 45,360 & 4,843 & 36,320 \\
\hline CT-body & 5,451 & 40 & 0.36 & 14.28 & 77,840 & 4,365 & 62,327 \\
\hline MR & 1,588 & 80 & 0.13 & 10 & 15,880 & 1,272 & 12,715 \\
\hline Total & 124,890 & & & & $2,491,661$ & 100,000 & $1,995,085$ \\
\hline
\end{tabular}


Table 4. Archive Components and Cost (Including Cost of Network Ports)

\begin{tabular}{lrlrrr}
\hline & & \multicolumn{3}{c}{ High-Speed Archive } \\
\cline { 3 - 5 } & & \multicolumn{1}{c}{ Component } & Cost (1990) & No. & Total \\
\hline 14" Two-drive Juke Box and software & $\$ 350,000$ & Creo Optical Tape and software & $\$ 200,000$ & 1 & $\$ 200,000$ \\
Decompressor (2) & $\$ 4,000$ & 1,2 GB r/w Optical disk & $\$ 2,500$ & 24 & $\$ 60,000$ \\
Compressor & $\$ 2,000$ & Disk-mux connection & $\$ 1,000$ & 24 & $\$ 24,000$ \\
& & Multiplexers & $\$ 10,000$ & 6 & $\$ 60,000$ \\
& & Decompressors & $\$ 2,000$ & 6 & $\$ 12,000$ \\
& & BISDN Ports & $\$ 10,000$ & 6 & $\$ 60,000$ \\
& & Compressor & $\$ 2,000$ & 1 & $\$ 2,000$ \\
LS Archive (1990) & $\$ 356,000$ & HS Archive (1990) & & $\$ 418,000$ \\
LS Archive (1995) & $\$ 178,000$ & HS Archive (1995) & & $\$ 209,000$ \\
LS Archive (2000) & $\$ 89,000$ & HS Archive (2000) & & $\$ 104,500$ \\
\hline
\end{tabular}

information for transmission to the hospital information system (HIS). We assume that both our film and PAC system interact with a RIS, so our analysis does not consider any RIS cost changes.

Archive technology. The advent of optical data devices that can store and quickly access vast quantities of image data is one of the critical technological advances that makes PACS feasible. Optical jukeboxes that allow access to 10 or more data platters, each holding up to 7 Gbyte are available as is an optical tape drive capable of storing 1 to 3 tbyte and scanned in under 1 minute. High-speed compression and decompression hardware is available that allows from $3: 1$ to 4:1 compression while mathematically guaranteeing the original image upon decompression. ${ }^{16}$ Such optical image storage devices, combined with conventional disk and main memory storage technology, can produce a two-layered or virtual memory storage system capable of reliably storing seven or more years of image data while still allowing very fast access to almost all patient data and images.

Low-speed archive. For low-speed networks, the archive need only output images at $5 \mathrm{Mbps}$ for it to keep up with the realistic data rates of the low-speed networks. Thus for a 100,000 procedure hospital a jukebox containing two 14-inch optical disk drives and room for 80 platters should be sufficient. Such a jukebox can store up to 560 Gbyte so with $3: 1$ to $4: 1$ compression it can provide on-line storage for a sizable portion of the total image database. The remaining portion of the database could be manually loaded as needed. The cost for such a jukebox is about $\$ 300,000$ in 1990 . Smaller jukeboxes and single platter drives are available to use with archives for smaller hospitals or partial PACS systems in larger ones.

High-speed archive. The archive for our hypothetical high-speed-network PACS must have both a high capacity long-term memory and very fast short-term memory. The long-term memory can consist of a three terabyte optical tape drive. This drive, which is currently available, has a seek time of about 30 seconds and a read rate of several megabits per second. With $3: 1$ or $4: 1$ compression, it could contain almost the entire 100,000 procedure hospital image database on a single tape. However, to avoid a great deal of tape swaping every six years during the tape changeover period, it would be reasonable to copy the most active folders onto a new tape every four years or so. The high-speed archive can be made even more reliable by adding a second optical tape drive located remotely at an additional cost. We do not consider this additional cost.

With about two terabytes of new information per year and 250 working days per year, an average hospital performing 100,000 procedures per year generates about 8 Gbyte of new images per working day. On average, the physicians will require the new study and existing images in the patient's image folder for about three or four days after the radiology procedure. Generally, an existing patient folder will contain about $4 \mathrm{MB}$ for each newly generated MB. ${ }^{15}$ Thus, each day eight GB must be read from long term memory, and $32 \mathrm{~GB}$ written. If we assume that the image folders must be keep in short-term memory on average for about 3.5 days, this means that short-term memory must contain about 112 Gbyte of uncompressed, or 28 Gbyte of compressed images.

In addition to providing sufficient capacity, the short-term memory must have a fast enough output rate to drive all workstations with sufficient response time. Our approach is to use 241.2 Gbyte read/write optical disk drives. By dividing each image among eight or more disk drives, the archive can output images at sufficient speed to provide adequate workstation response time. We have calculated through static analysis using posson distributions that this archive should be able to keep up with a worse case load of all workstations simultaneously performing scroll operations every two seconds. Table 4 summarizes the components and costs for potential configurations of LS and HS archives.

\section{REFERENCES}

1. Parrish D, Beard DV, Kilpatrick KE, et al: Operational modeling for PACS: How do we decide if its cost effective? SPIE Medical Imaging III 1093:457-465, 1989

2. Dwyer III SJ, Templeton AW, Martin NL, et al: The cost of managing digital diagnostic images. Radiology 144 : 312-318, 1982

3. Vanden Brink J, Cywinski J, Szerlag CT: Cost analysis of present methods of image management. SPIE Medical Imaging 767:758-764, 1987

4. Seshadri SB, Arenson RL, et al: Cost-savings associated with a digital radiology department: A preliminary study in 9th Conference on Computer Applications in Radiology RISC/ACR. Hilton Head SC, RISC/ACR, 1988

5. Mun SK, Benson J, Welsh C, et al: Baseline study of 
radiology services for the purpose of PACS evaluation. SPIE Medical Imaging II 914:978-987, 1988

6. Pizer SM, Beard DV: Medical image work stations: Functions and implementation. J Digital Imag 2:185-193, 1989

7. Beard DV: Designing a radiology workstation: Focus on navigation during the interpretation task. University of North Carolina, Chapel Hill, Department Computer Science Technical Report, J Digital Imag 1990 (submitted)

8. Vortech Data Inc: Product specification. Reston, VA, 1989

9. CREO Products Inc: Product specifications. Burnaby, British Columbia, Canada, 1989

10. Salwen H: High speed networking: The next ten years. Telecommunications 23:47-52, 1989

11. Bellcore: TA-772 metropolitan area-network generic framework system requirement in support of switched multimegabit data service. 1988

12. Kohli J: Medical imaging applications of emerging broadband networks. IEEE Communications Magazine, 816,1989

13. Ultra-Network Technologies: UltraNet Technical Specifications. San Jose, CA, 1988

14. Beard DV, Cromartie R, Creasy J, et al: Experiment comparing image-locating on film and the FILM-PLANE radiology workstation. SPIE Medical Imaging II 914:933937,1988

15. Naylor Company: Probable Evolution of Pictorial Archiving and Communication Systems for Radiology Report 336-1, Version 3.0. A.F. Naylor Company, Cleveland, OH, 1989

16. Roos P, Viergever MA, Van Dijke MCA, et al: Reversible intraframe compression of medical images. IEEE Trans Med Imag 7:328-336, 1988 